\title{
Sextus Empiricus, scepticisme et philosophie de la vie quotidienne
}

\section{Stéphane Marchand}

\section{(2) OpenEdition}

1 Journals

Édition électronique

URL : https://journals.openedition.org/philosant/366

DOI : 10.4000/philosant.366

ISSN : 2648-2789

Éditeur

Éditions Vrin

\section{Édition imprimée}

Date de publication : 24 novembre 2015

Pagination : 91-119

ISBN : 978-2-7574-1141-4

ISSN : 1634-4561

\section{Référence électronique}

Stéphane Marchand, «Sextus Empiricus, scepticisme et philosophie de la vie quotidienne », Philosophie antique [En ligne], 15 | 2015, mis en ligne le 01 novembre 2018, consulté le 02 décembre 2022. URL : http://journals.openedition.org/philosant/366 ; DOI : https://doi.org/10.4000/philosant. 366

\section{(c) (i) ()}

Creative Commons - Attribution - Pas d'Utilisation Commerciale - Pas de Modification 4.0 International - CC BY-NC-ND 4.0

https://creativecommons.org/licenses/by-nc-nd/4.0/ 
SEXTUS EMPIRICUS, SCEPTICISME

ET PHILOSOPHIE DE LA VIE QUOTIDIENNE

Stéphane MARCHAND

IHPC (UMR 5037)

RÉSUMÉ. Quel rôle joue le concept de vie quotidienne dans le scepticisme de

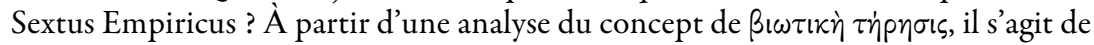
faire apparaitre, d'une part (i) que la vie quotidienne, par opposition à la vie philosophique, est un fait empirique qui permet au sceptique d'agir sans pour autant avoir d'opinions et d'autre part que (ii) la vie quotidienne est une valeur qui donne sens à la philosophie sceptique. Bien que ces deux approches paraissent contradictoires, le but de cet article est de montrer que le scepticisme philosophique proposé par Sextus les rend compatibles.

SUMMARY. What role does the notion of 'everyday life' play in Sextus Empiricus's skepticism? On the basis of an analysis of the concept of $\beta 1 \omega \tau \tau x \dot{\eta} \tau \eta \dot{\rho} \eta \sigma \mathrm{r}$, this paper purports to show (i) that everyday life, as opposed to 'philosophical life', is an empirical fact that allows the Pyrrhonist to act without holding beliefs, and (ii) that everyday life is a genuine value of the Pyrrhonian philosophy. Even though these two theses may seem contradictory, the aim of the present paper is to show that Sextus's philosophical skepticism make them compatible. 

Le but de cet article est de clarifier le sens et la fonction du concept de

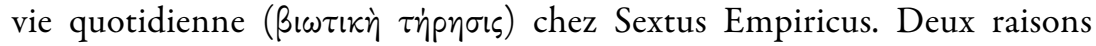
poussent à cela : d'une part le fait que ce concept joue un rôle important, sinon fondamental, dans sa conception du scepticisme; d'autre part le fait que la vie quotidienne constitue un problème philosophique en soi. Que l'on considère la quotidienneté comme le lieu d'une perte de l'authenticité, ou au contraire que l'on utilise la vie quotidienne pour critiquer les erreurs de l'abstraction, la vie quotidienne est un véritable objet philosophique à interroger. Henry Lefebvre, par exemple, a montré dans sa Critique de la vie quotidienne la difficulté de parler du haut ou au nom de la vie quotidienne. Il remarque que la conception philosophique de la vie quotidienne est le plus souvent une construction théorique qui risque toujours de manquer la vie quotidienne elle-même ${ }^{1}$. Et, souligne-t-il, les philosophes et les intellectuels ne sont peut-être pas les mieux placés pour saisir la vie quotidienne en elle-même, tout occupés qu'ils sont par leur volonté de vérité ou par leur quête d'authenticité. En réalité, il est difficile de déterminer où se trouve la vie quotidienne et quels individus, quelles expériences doivent être choisis comme représentatifs de la vie quotidienne.

Il faut commencer par préciser que l'expression « vie quotidienne » ne désigne pas dans le néo-pyrrhonisme un concept complètement stabilisé au sens où, par exemple, il renverrait à une expression lexicalisée et utilisée sys-

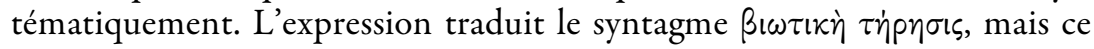
dernier ne se rencontre chez Sextus qu'en quatre occurrences, toutes localisées dans les Esquisses Pyrrhoniennes ${ }^{2}$ (désormais $P H$ ), alors que - telle est du moins la thèse de cet article - l'idée de « vie quotidienne » joue un rôle majeur dans la conception sextienne du néo-pyrrhonisme et constitue un de ses problèmes fondamentaux. Ce concept, en réalité, est constitué

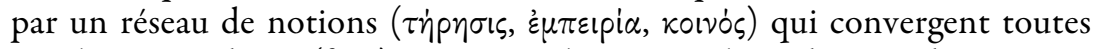
vers la notion de vie (Bios). La compréhension et la traduction de ce réseau 
conceptuel par «vie quotidienne» peuvent elles-mêmes se discuter : si l'on compare les différentes traductions de PH I 23-24 où l'expression apparaît, on voit d'ailleurs qu'il y a une hésitation entre l'idée de «vie commune $\gg^{3}$, de $\ll$ vie ordinaire $»^{4}$ et de $\ll$ vie quotidienne $»^{5}$.

Le but de cet article est double : il s'agit, d'une part, d'étudier comment Sextus Empiricus utilise ce concept qui ne désigne pas toute forme de vie, mais une vie particulière, la vie ordinaire par opposition à la vie philosophique, celle qui se donne à voir empiriquement tous les jours dans les faits et gestes quotidiens des hommes qui nous entourent, une vie donc qui, pour cette raison, peut être désignée comme la vie quotidienne. Cette vie s'impose donc comme un fait observable empiriquement. D'autre part, il s'agit de se demander comment ce fait de la vie quotidienne peut devenir une valeur dans le cadre d'une philosophie sceptique : comment concevoir une philosophie de la vie quotidienne ${ }^{6}$ ?

\section{La vie quotidienne comme fait empirique}

\subsection{La vie quotidienne comme une réponse à l'objection de l'ả $\pi \rho \alpha \xi i \alpha$}

Avant toute chose, il est nécessaire de rappeler que la nature fondamentalement critique du scepticisme empêche Sextus d'énoncer des affirmations qui pourraient être interprétées comme des opinions ou des thèses. Par conséquent, Sextus ne peut pas faire une théorie de la vie quotidienne; pour comprendre le sens de ce concept, il faut explorer le réseau des

3 Cf. Estienne 1562 : «ea, quae ad vitam communem pertinent, observantes vivimus », Tescari 1926 : «viviamo senza dogmi, osservando le norme della vita comune ».

4. Cf. Pappenheim 1877 : «leben wir gemäss der Beobachtung des (gewöhnlichen) Lebens ansichtslos », Bury $1933:$ : we live in accordance with the normal rule of life », Mates 1996 : « we live without beliefs but in accord with the ordinary regimen of life 》.

5. Annas et Barnes 1994: «we live in accordance with everyday observance, without holding opinions », Pellegrin 1997 : « nous vivons en observant les règles de la vie quotidienne ». Pour être complet, il faut mentionner la traduction de Gallego Cao et Muñoz Diego 1993 qui interprètent - à tort selon moi - la formule comme une référence à la vie au sens physiologique du terme: «vivimos sin dogmatismos, en la observancia de la exigencias vitales $\gg$.

6. La vie quotidienne chez Sextus a été étudiée par Grgić 2011 ; on trouve aussi des analyses sur ce sujet dans Barnes 1988 et Spinelli 2005, chap. 6 ; la critique a été occupée avant tout par le problème de la séparation entre le scepticisme philosophique et la vie quotidienne (désigné dans la littérature secondaire - majoritairement anglophone - comme le problème de l'insulation of skepticism), c'est-à-dire la question de savoir dans quelle mesure le scepticisme proposé par Sextus est compatible avec la vie quotidienne, $c f$. Bett 1993; l'approche de la présente étude est différente puisqu'elle consiste à se demander dans quelle mesure la vie quotidienne peut constituer un modèle pour le scepticisme. 
arguments et contre-arguments sceptiques afin de voir comment il apparaît et quelles sont ses implications.

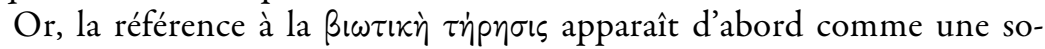
lution pragmatique à l'objection de l'ả $\pi p \alpha \xi i \alpha$. Le texte principal qui expose cette solution vient des Esquisses Pyrrhoniennes I 23-24:

Donc en nous attachant aux phénomènes nous vivons sans opinions selon ce qui est observé dans la vie quotidienne puisque nous ne pouvons pas être complètement inactifs. Il semble que ce qui est observé dans la vie quotidienne ait quatre aspects : l'un consiste dans la conduite de la nature, un autre dans la nécessité de nos affects, un autre dans la tradition des lois et des coutumes, un autre dans l'apprentissage des arts ${ }^{7}$.

Bien sûr, il est toujours possible de lire cette réponse, sinon comme une réponse purement ad hominem, du moins comme une réponse strictement liée à cette objection dogmatique qui n'exprimerait rien de la position sceptique elle-même ${ }^{8}$. Mais ma thèse est, au contraire, que cette réponse exprime une position cruciale pour Sextus, et que la référence à la vie quotidienne est constitutive de la position néo-pyrrhonienne?.

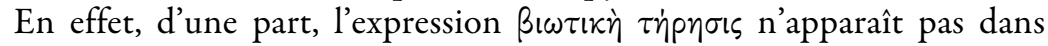
d'autres corpus de la littérature grecque ${ }^{10}$. Cela mérite d'être souligné d'autant plus que, par ailleurs, la plupart des concepts utilisés par les sceptiques sont empruntés à la philosophie dogmatique. Cette expression apparaît donc seulement chez Sextus et uniquement dans les Esquisses, bien qu'il y ait, comme on le verra, des équivalents dans les autres œuvres de Sextus ${ }^{11}$,

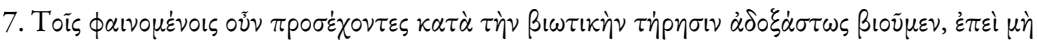

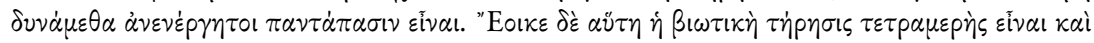

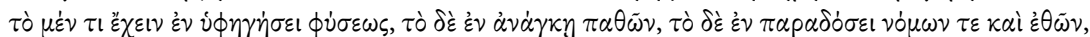
$\tau \grave{\partial} \delta \dot{\varepsilon} \dot{\varepsilon} \nu \delta \delta \delta \alpha \sigma \alpha \lambda \lambda \dot{\alpha} \tau \varepsilon \chi \chi \nu \tilde{\omega} v$. Pour les Esquisses Pyrrhoniennes, sauf mention contraire, j'utilise, en la modifiant à l'occasion, la traduction de Pellegrin 1997.

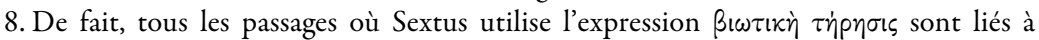
l'objection de l'inactivité, sauf $P H$ III 235.

9. Un signe de l'implication personnelle de Sextus dans cette réponse est d'ailleurs l'usage de la première personne $\beta$ เоच̃ $\mu \varepsilon v$ dans ce texte.

10. À l'exception, ce qui n'est en rien fortuit, de termes proches utilisés par Galien dans des textes liés à la tradition médicale empirique, $c f$. infra $\mathrm{n}$. 40. Il est significatif, en outre, que ce motif n'apparaisse pas non plus dans la tradition néo-académicienne qui, pourtant, a dû affronter le même type d'objection, mais qui répond avec l'eủ $\lambda$ óyov d'Arcésilas ( $c f$. Ad-

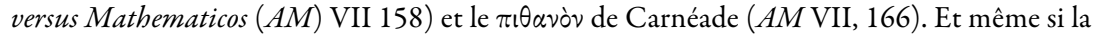
solution d'Arcésilas est fortement liée à la nature, il s'agit d'une autre forme de réponse que la référence pyrrhonienne au ßı́s. Cf. Striker 1980; Ioppolo 1986 ; Bett 1989; Ioppolo 2009; Vogt 2010; Obdrzalek 2012 pour l'analyse des réponses néo-académiciennes à l'objection de l'inactivité.

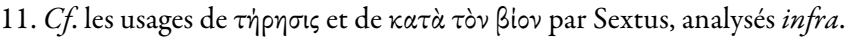


ainsi qu'au livre IX de Diogène Laërce, lequel, dans des termes proches, affirme que « les sceptiques disent qu'il [le sceptique] aura la possibilité de vivre en s'abstenant de toute enquête sur les questions dogmatiques, mais non sur celles qui intéressent les affaires de la vie ordinaire et les observations empiriques $\gg^{12}$. La présence de ce motif dans la doxographie de Diogène laisse d'ailleurs penser que, dans la mesure où il utilise des sources indépendantes de Sextus, ce concept est lié à la tradition néo-pyrrhonienne en général et n'est pas uniquement une invention de Sextus.

Par conséquent, même si ce concept apparaît dans un mouvement dialectique en réponse à une objection, la vie quotidienne peut être considérée comme un concept authentiquement sceptique, clairement attesté dans la tradition néo-pyrrhonienne ${ }^{13}$, utilisé pour décrire une particularité de la position sceptique; nous pouvons donc suivre ici Filip Grgić lorsqu'il dit que $\ll$ Sextus sees the skeptics as champions and supporters of ordinary life $\gg^{14}$.

En ce qui concerne le sens de l'argument, il est en apparence assez simple : à ceux qui objectent que le sceptique ne peut agir sans opinion $(\dot{\alpha} \delta 0 \xi \dot{\alpha} \sigma \tau \omega \varsigma)$, Sextus répond que l'action du sceptique est possible et consiste à faire ce que tout le monde fait dans la vie quotidienne en quelque sorte instinctivement, du moins sans y penser plus que cela, sans mobiliser aucune opinion dogmatique. Cette réponse est liée à l'explication systé-

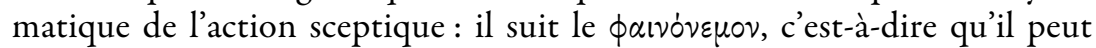
agir parce qu'il a des sensations et des pensées qui peuvent lui donner un critère d'action ou, comme il le dit parfois, des « critères issus de la vie »

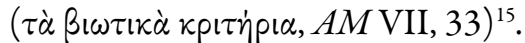

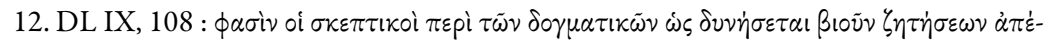

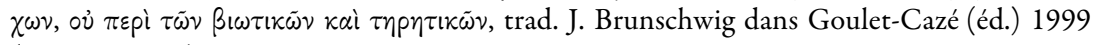
(trad. modifiée).

13. Si le motif de la vie quotidienne est lié aux sources les plus tardives sur la tradition néo-pyrrhonienne (Sextus et Diogène), on peut néanmoins relever quelques traits qui pour-

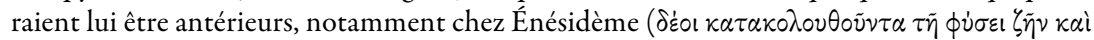
$\tau \tau^{i} \varsigma$ है $\theta \varepsilon \sigma \iota:$ 《il faut vivre en suivant la nature et les coutumes », Aristoclès apud Eusèbe, Prép. év. XIV 18, 20 si l'on suit les hypothèses de Chiesara 2001 pour qui Aristoclès s'appuyait sur Énésidème) et chez Timon dans la référence à la $\sigma u v \eta \dot{\theta} \theta \varepsilon \alpha, c f$. DL IX, 105. F. Decleva Caizzi pense néanmoins que cette dernière référence est sans rapport avec la notion néo-pyrrhonienne de vie quotidienne, $c f$. Decleva Caizzi 1981 p. 238 ; pour un rapprochement, voir cependant Brochard 1887 p. 73.

14. Grgić 2011, p. 69.

15. Pour la distinction entre critère de vérité et critère d'action, cf. $P H$ I 21 ; voir aussi $P H$ II, 14 : « on appelle critère à la fois ce par quoi, disent-ils, on juge de l'existence et de la

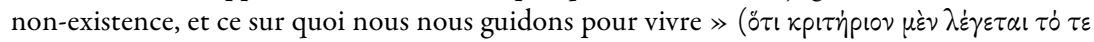

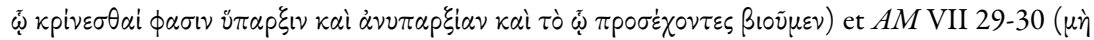

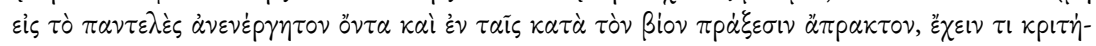

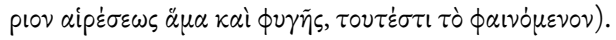


En première analyse « vivre sans opinion selon ce qui est observé dans la vie quotidienne » signifie donc vivre comme tout le monde, c'est-à-dire comme la plupart des gens, vivre, en quelque sorte, selon un mode de vie ordinaire, celui que nous avons sous les yeux tous les jours. Selon cette première analyse, le sens de l'argument qui fait référence à la vie quotidienne consiste donc à répondre aux dogmatiques non seulement que le sceptique peut agir, mais que tout le monde agit la plupart du temps de cette façon, à savoir sans avoir d'opinion.

\subsection{La vie, la vie ordinaire et la vie quotidienne}

Cette caractérisation est cependant insuffisante. Bien que le sens de la réponse à l'objection de l'ả $\pi \rho \alpha \xi i \alpha$ paraisse clair, il reste quelques problèmes. Il y a, d'une part, un problème de traduction qui dénote un problème de

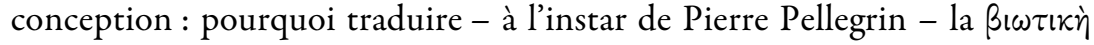
$\tau \dot{p} p \eta \sigma \iota s$ par « vie quotidienne » ? Avant de se concentrer sur le terme de $\tau \dot{\eta} p \eta \sigma \varsigma$, , il convient d'élucider la nature de la vie dont il est question ici : en quel sens la vie dont parle Sextus renvoie-t-elle à la quotidienneté plutôt qu'à la naturalité ou à la normalité ? Encore une fois, la notion de vie quotidienne n'est nullement stabilisée chez Sextus et il est nécessaire de reconstruire le sens de ce concept en essayant de clarifier le réseau notionnel qu'il mobilise autour du ßios.

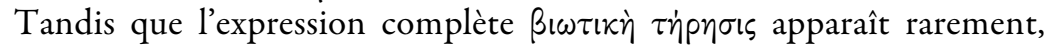
Sextus utilise fréquemment la référence à la vie sans autre qualification,

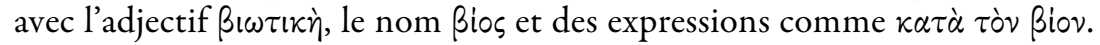
Cette variatio renvoie souvent, cependant, à la même fonction que la référence à la vie quotidienne. La référence à la vie permet en effet d'opposer au fonctionnement dogmatique un autre modèle d'action. C'est le cas notamment en $P H$ II 102 lorsque Sextus décrit l'usage accepté par les sceptiques du signe commémoratif «parce qu'il est considéré comme crédible par la vie quotidienne $\gg^{16}$. Non seulement nous faisons tous les jours, selon Sextus, l'expérience qui consiste à raisonner en allant du signe à ce qu'il signifie lorsque le lien entre les deux est évident, mais tous les jours nous voyons les hommes se fier à cette association. Alors, dit Sextus dans le même paragraphe, le sceptique « non seulement ne s'oppose pas à la vie quotidienne, mais il prend même sa défense en donnant son assentiment, sans soutenir d'opinions, à ce que la vie quotidienne considère comme crédible $\gg^{17}$. De même lorsque Sextus parle des « besoins de la vie »( $\chi p \varepsilon i \alpha \varsigma, P H$ II 246) ou des «expériences de la vie » $(\tau \tilde{\omega} \nu \beta \iota \omega \tau \iota k \tilde{\omega} \nu \varepsilon \dot{\mu} \mu \pi \varepsilon ı$ i $\tilde{\omega} \nu$,

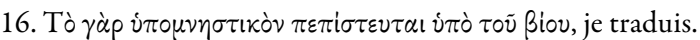

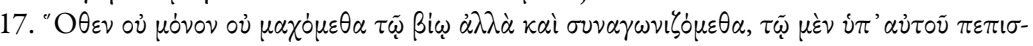

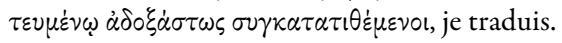


PH II 258), il ne s'agit pas de prendre en compte toutes les expériences de vie, ni même toutes les expériences de vie humaine, mais de prendre le parti d'une certaine forme d'expérience de vie, celle qui se manifeste dans la vie quotidienne, par opposition à une vie particulière, la vie dogmatique, qui repose sur la croyance en des opinions ${ }^{18}$.

Cela ne veut pas dire que lorsque Sextus utilise le terme ßios, il fasse toujours référence à la vie quotidienne ${ }^{19}$, mais il paraît indéniable qu'il existe chez Sextus un usage particulier du terme qui renvoie à une certaine expérience de la vie, celle de la vie commune (cf. PH I 237: ó ßios ó kovvós) et qui désigne une certaine forme de vie : la vie simple, ordinaire, commune au sens où elle partagée par tous et donc banale.

Si Sextus ne donne guère d'éléments supplémentaires pour comprendre positivement cette vie, il est plus prolixe pour dire ce à quoi elle s'oppose. La vie quotidienne désigne ce qui n'est pas la vie théorétique : la vie dogmatique ou encore la vie philosophique ${ }^{20}$. Le $\$ 165$ du Contre les Moralistes ( $A M \mathrm{XI})$ constitue en ce sens un témoignage de choix :

le sceptique ne vit pas selon un raisonnement philosophique - il est inactif, en effet, pour autant qu'il est question de ce dernier - mais selon ce qui est

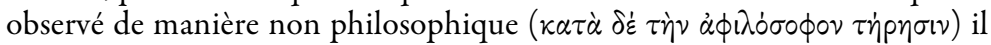
peut désirer certaines choses et en fuir d'autres ${ }^{21}$.

Dans le même contexte d'une réponse à l'objection de l'ả $\pi \rho \alpha \xi i \alpha$, Sextus utilise l'expression $\kappa \alpha \tau \dot{\alpha} \delta \dot{\varepsilon} \tau \dot{\eta} \nu \grave{\alpha} \phi i \lambda \dot{\sigma} \sigma \circ \phi o v \tau \eta \dot{\eta} p \eta \sigma \tau$ comme un équivalent du

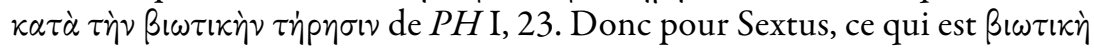

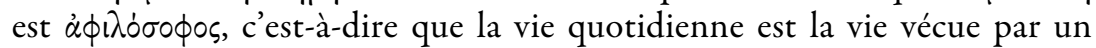
non-philosophe, la vie vécue par ceux qui agissent sans référence à une théorie générale ou à un dogme sur la nature réelle des choses. La question de savoir si, selon Sextus, tous les non-philosophes et plus généralement tous les « hommes ordinaires » vivent sans opinion reste ouverte, et nous verrons dans la deuxième partie que Sextus émet quelques doutes à ce sujet, notamment dans le domaine éthique ${ }^{22}$; il n'en reste pas moins que lorsque Sextus s'occupe de proposer un modèle d'action non dogmatique, le plus souvent il exprime ce modèle négativement en l'opposant à la vie philosophique.

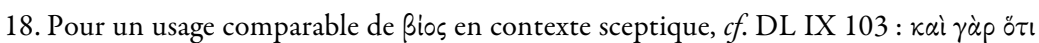

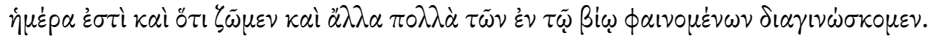

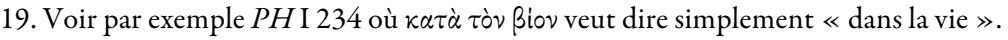

20. Cf. Barnes 1997, p. 79 sqq.

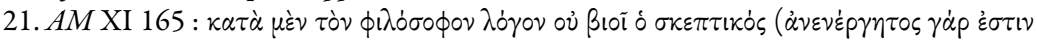

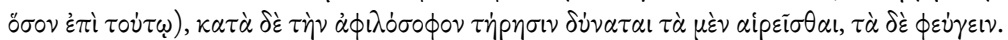

22. Comme nous le verrons, il peut arriver que Sextus dénonce le dogmatisme de certains « hommes ordinaires $\gg(o i \quad i \delta i \tilde{\omega} \tau \alpha l)$. 
La première analyse de la vie quotidienne doit donc être complétée : il s'agit de vivre comme un «homme ordinaire », c'est-à-dire sans s'embarrasser de la théorie ou de la philosophie. Pour autant, à ce niveau d'ana-

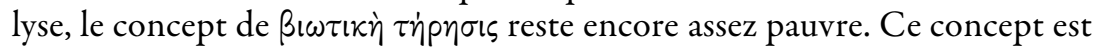
défini négativement tout au plus : il s'agit d'une référence négative à une vie sans philosophie, avec l'affirmation, sans véritable justification, que les gens ordinaires vivant une vie quotidienne font mieux que les philosophes. Telle quelle cette détermination n'est pas suffisante; il faut justifier le modèle épistémique utilisé pour penser cette vie quotidienne : d'où provient cette représentation de la vie quotidienne ? À quelle expérience fait-elle référence ? Après tout, il y a plusieurs façons de concevoir la vie quotidienne. Ce concept n'a rien de neutre, il constitue même un objet philosophique construit, sur lequel il peut y avoir un certain nombre de désaccords. La vie quotidienne fait l'objet de projections, de constructions philosophiques et donne matière au dogmatisme. Bref, sans justification de l'origine épistémique de ce concept, il est tout à fait possible d'objecter à Sextus Empiricus qu'il dispose d'un concept dogmatique de vie quotidienne, ce qui met en péril son scepticisme. Pour cette raison, il faut mettre à jour les fondements épistémiques de son concept de vie quotidienne.

\subsection{La vie quotidienne comme méthode : l'empirisme}

Il serait erroné de penser qu'il s'agit là d'un angle mort de la pensée de Sextus. Ce dernier a lui-même donné une justification du modèle épistémique qui préside à sa conception de la vie quotidienne. Pour la com-

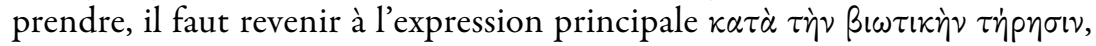
et plus précisément à la partie de cette expression dont il n'a pas encore été rendu compte : la $\tau \dot{\eta} p \eta \sigma \iota \zeta$, l'observation. La vie quotidienne n'est pas simplement une référence négative à la vie non philosophique, il s'agit aussi du résultat d'une méthode d'observation empirique. Il semble donc que la formule de PH I 23, « vivre selon ce qui est observé dans la vie quotidienne », puisse être encore précisée : non seulement il s'agit de vivre comme tout un chacun, non seulement il s'agit de vivre sans avoir recours à la philosophie, mais il s'agit de suivre un modèle d'action qui peut lui-même être observé dans la vie quotidienne. La vie quotidienne doit être, à la lettre, observée, c'est-à-dire suivie, mais c'est parce qu'elle se donne à voir empiriquement qu'elle peut être suivie : parce qu'elle est empiriquement observable, elle peut être observée pratiquement. L'empirisme désigne donc ici le processus épistémique qui permet de disposer d'un exemple d'action qui peut devenir un modèle : l'action que l'on voit au quotidien. Pour cette raison, la tra-

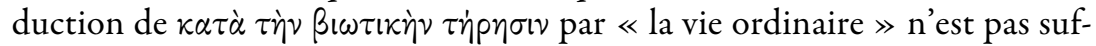
fisante : il ne s'agit pas seulement de la «vie ordinaire en tant qu'elle s'opposerait à la vie philosophique, il s'agit de la «vie quotidienne », de 
cette vie que nous avons sous les yeux tous les jours, que nous avons le loisir d'observer, d'imiter afin d'apprendre à vivre. Cette vie n'est pas entièrement sans opinion, mais le modèle empirique mobilisé permet précisément de comprendre quel type d'opinion le sceptique cherche à éviter : non pas toute forme d'opinion, mais l'opinion forte sur la nature des choses, c'est-àdire la forme d'opinion propre à la pratique scientifique ou théorique, en laissant possible le fait d'avoir des opinions faibles, propres au sens commun ou à la vie quotidienne ${ }^{23}$.

\subsubsection{Le chapitre des Esquisses sur les sophismes (PH II 229-259).}

Pour éclairer cette dimension empiriste de la démarche de Sextus, il peut être utile de s'arrêter sur un chapitre des Esquisses. Le but du chapitre 22 du deuxième livre des Esquisses est de montrer comment la voie sceptique peut aussi éviter les sophismes :

Nous, en partant, sans soutenir d'opinions, des observations de la vie quotidienne, nous évitons ainsi les arguments fallacieux, alors que les dogmatiques n'auront pas la possibilité de distinguer le sophisme du raisonnement qui semble être proposé comme il faut, s'il est vrai qu'ils doivent décider dogmatiquement que la forme du raisonnement est concluante et que ses prémisses sont vraies, ou que ce n'est pas le $\operatorname{cas}^{24}$.

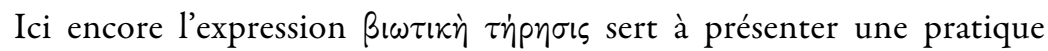
sceptique par opposition à celle du dogmatique en général, et plus précisément ici par opposition à celle du dialecticien. Sextus montre comment le sceptique peut se sortir des embrouillaminis des dialecticiens en s'appuyant sur la vie quotidienne puisque, selon Sextus, le sens commun et la vie commune sont plus efficaces que la dialectique stö̈cienne pour se débarrasser des sophismes ${ }^{25}$.

Mais ce chapitre 22 ne se contente pas de répéter cette position; il développe le modèle de la vie quotidienne en insistant sur sa dimension empirique. Ce faisant, il pose un certain nombre de questions qu'il faut ici rapidement aborder. Tout d'abord, le chapitre prend parti dans un domaine - la dialectique - où certains philosophes présentent leur activité

23. Cf. $P H$ I 13. Ce problème est au cœur de la polémique des années $80, c f$. les articles rassemblés dans Burnyeat et Frede 1997; mon interprétation est globalement proche de celle de Frede 1997, même si, comme nous allons le voir infra, la vie quotidienne et le sens commun peuvent comporter des croyances fortes, notamment dans le champ moral.

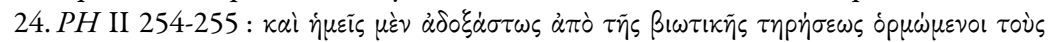

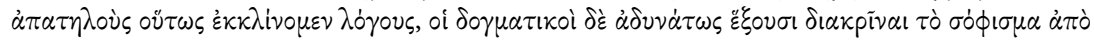

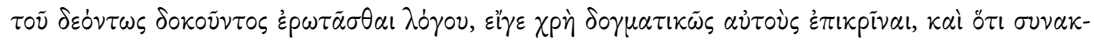

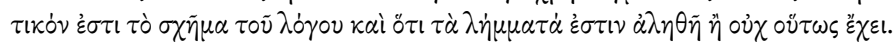

25. Sur ce chapitre, voir Spinelli 2009. 
comme utile « en prétendant donner des conseils dans les incertitudes de

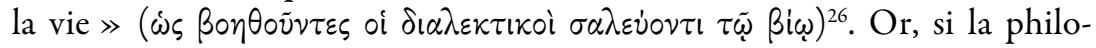
sophie sceptique est une «philosophie de la vie quotidienne », elle doit pouvoir rendre compte de cette situation problématique où la vie semble à l'origine même de nos problèmes. Ensuite, si la solution de Sextus consiste bien à montrer qu'il est possible, tout en restant sceptique, de développer une technique et une spécialisation particulières pour résoudre les problèmes posés par les faux raisonnements, il faut se demander s'il ne nous propose pas ici une technique qui nous éloigne de la sagesse de la vie quotidienne.

Pour commencer, il faut remarquer que ce n'est pas Sextus qui affirme qu'il y a des « incertitudes de la vie » mais le $\$ 229$ reprend ici une position dogmatique comme le marque clairement le $\omega \varsigma$. Sextus ne concède en rien l'utilité de la dialectique dogmatique, mais montre au contraire que « la résolution de tous les sophismes qui semblent pouvoir être spécifiquement réfutés par la dialectique est inutile » ( $P H$ II, 236). Il s'agit de tous les faux problèmes, c'est-à-dire des problèmes purement théoriques sans incidence pour la vie quotidienne ${ }^{27}$. Selon un schéma que nous retrouverons plus tard, ce n'est pas la vie elle-même qui pose problème, mais bien les erreurs de raisonnement que nous faisons sur elle. Il n'en reste pas moins que les sceptiques, comme tous les hommes, peuvent se retrouver devant des problèmes logiques qui demandent à être résolus parce qu'ils touchent des domaines nécessaires à la vie. Il s'agit notamment, selon les exemples pris par Sextus, des erreurs de raisonnement en médecine, où le raisonnement débouche sur une décision et une action dont la vie, précisément, dépend.

Cette réponse permet d'aborder la seconde objection : dans ce cas, ce n'est pas la vie toute seule, et encore moins la « vie quotidienne » qui semble suffisante pour résoudre ces problèmes. Et Sextus dit que ces sophismes qui posent de vrais problèmes sont réfutés par « ceux qui, en chaque art, ont saisi la connexion entre les choses $\gg^{28}$, c'est-à-dire les hommes de l'art, les techniciens, et en l'occurrence. dans l'exemple de PH II 237-240, les médecins. Cette réponse est-elle contradictoire avec le modèle de la vie quotidienne et l'opposition précédemment dessinée entre vie et philosophie ? Il ne semble pas que cela soit le cas puisque précisément le modèle médical, et plus généralement le modèle de $\tau \dot{\varepsilon} \chi \chi \eta ~ u t i l i s e ́$ par Sextus, s'appuie sur une approche pragmatique fondée sur les seules $\tau \dot{\varepsilon} \chi \nu \alpha \mathrm{l}$ utiles ${ }^{29}$. Ces tech-

26. PH II 229, trad. Pellegrin modifiée.

27. Cf. les exemples développés au livre II. \$230-235 et 241-245.

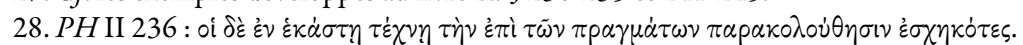

29. Sur l'utilité comme critère pour faire des distinctions entre les arts, cf. Barnes 1988 p. 65 et Spinelli 2010 p. 258-259. 
niques s'enracinent dans des pratiques séculaires, répétées, qui se sont sédimentées dans l'expérience et la mémoire, et qui proviennent de la vie quotidienne. Le fait que certains hommes soient plus habiles que d'autres dans ces domaines empiriques n'est pas suffisant, selon Sextus, pour détacher ces pratiques du sol de la vie quotidienne. Alors, certes, ces hommes qui saisissent « la connexion entre les choses » ne sont plus tout à fait des « hommes ordinaires » au sens où ils ont développé une spécialisation qui les rend plus compétents, notamment pour débusquer des sophismes dans leur domaine de compétence, mais pour autant cette compétence n'en est pas moins enracinée dans la vie quotidienne; et en ce sens ils restent du côté de la vie par opposition à la théorie.

Cette critique de l'approche théorique des problèmes n'est pas réservée à la logique, puisque l'on peut trouver des usages de l'expression $\kappa \alpha \tau \dot{\alpha} \tau$ còv Biov à chaque fois que Sextus montre que nous pouvons faire quelque chose sans avoir d'opinion, juste en utilisant un critère d'action et non un critère de vérité $e^{30}$. Dans le même esprit, ces passages tendent à montrer que la vie

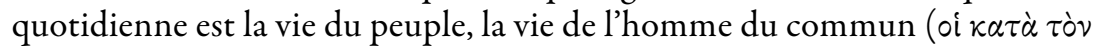
Biov $\alpha \nu \theta p \omega \pi \circ \mathrm{l})$, qui n'est pas perturbée par des arguments philosophiques. C'est le cas notamment lorsqu'il s'agit de traiter les arguments contre le mouvement: les gens normaux, ordinaires, ont démontré pragmatiquement la vacuité de ces arguments, en vivant, par opposition aux philosophes qui sont toujours à la recherche de réponses aux arguments de Diodore Cronos ${ }^{31}$. Dans le même registre et avec des termes similaires, Sextus utilise la vie quotidienne comme un modèle pour penser la meilleure façon de parler grec, par opposition aux grammairiens qui proposent une théorie de l'hellénisme ${ }^{32}$.

Mais ce qui fait l'intérêt capital de ce chapitre vient de ce qu'il donne une description du raisonnement empirique qui préside à cet usage de la vie quotidienne :

il est, en effet, suffisant, je pense, de vivre en suivant l'expérience, sans opinions, selon les observations et les préconceptions communes, suspendant

30. Cf. $P H$ I 21 : « en nous y attachant [scil. au critère d'action], dans le cours de notre vie, nous ferons telles choses, et ne ferons pas telles autres, et c'est celui-là dont nous parlons

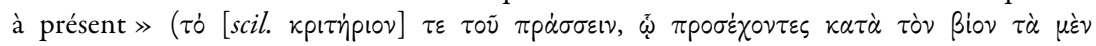
$\pi p \dot{\alpha} \sigma \sigma \circ \mu \varepsilon \nu \tau \dot{\alpha} \delta^{\prime} \circ \tilde{u}^{\prime}$.

31. PH II 244. Voir aussi II 257 ; $A M$ XI 68.

32. $A M$ I 179 : « ceux qui veulent parler correctement doivent s'appuyer sur l'observation simple et non technique qui suit la vie quotidienne ainsi que sur l'observation qui

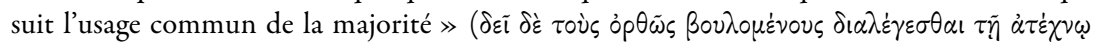

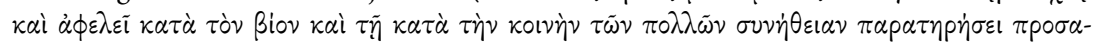
$\nu \dot{\varepsilon} \chi \varepsilon \varepsilon v$, je traduis). Sur ce texte, $c f$. Dalimier 1991. 
notre assentiment sur les assertions provenant des superfluités dogmatiques qui sont tout à fait en dehors de la vie ${ }^{33}$.

Il importe une nouvelle fois de souligner que ce passage ne peut pas être interprété comme un texte ad hominem dans lequel Sextus aurait emprunté une théorie dogmatique afin de produire une équipollence d'arguments : ce texte se présente comme une exposition de la position personnelle de Sextus. Il s'agit d'un commentaire de la réfutation pragmatique des arguments de Diodore Cronos sur le mouvement, juste après le récit de l'anecdote qui montre le médecin Hérophile appliquant l'argument contre le mouvement afin de convaincre Diodore que son épaule n'est pas luxée : «de sorte que le sophiste le supplia de laisser de côté les raisonnements de ce genre et d'entreprendre le traitement que la médecine prescrivait comme adapté à son cas $\gg(P H \text { II 245) })^{34}$. Même si Hérophile n'est pas un médecin empirique, nous savons qu'il est lié à une approche empirique de la médecine avant même la constitution d'une école médicale empirique ${ }^{35}$. Donc, même si par ailleurs Sextus a exprimé des réserves vis-à-vis de l'école empirique de médecine ${ }^{36}$, il semble apprécier les caractéristiques empiriques propres à la littérature médicale empirique, et partage avec les empiriques le même antidogmatisme et le même appel au sens commun : les deux mouvements se rejoignant d'ailleurs autour de l'usage de la $\tau \eta p \dot{\eta} \sigma \varsigma^{37}$.

Il y a donc une caractéristique commune entre la vie sans opinion ( $\dot{\alpha} \delta \circ-$ $\xi \dot{\alpha} \sigma \tau \omega \varsigma)$ et la vie «par expérience », દ́ $\mu \pi \varepsilon i p \omega \varsigma$. Et cette précision est importante parce qu'elle établit un lien entre la référence à la vie quotidienne

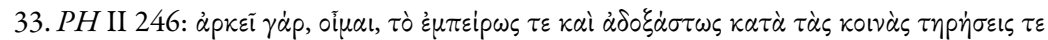

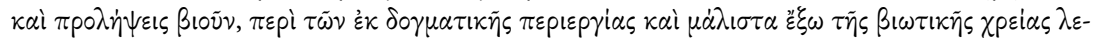
$\gamma \circ \mu \dot{\varepsilon} \nu \omega \nu \dot{\varepsilon} \pi \dot{\varepsilon} \chi 0 \nu \tau \alpha \zeta$.

34. Sur la figure de Diodore chez Sextus, $c f$. Sedley 1977.

35. La thèse selon laquelle Hérophile était pyrrhonien est défendue par Kudlien 1964 et a été réfutée par Von Staden 1989. Von Staden montre que « not only are Herophilus statements of method therefore incompatible with the Empiricists'; the repeated methodological attacks by the Empiricists on Herophilus and his followers from the third to the first century B.C. bear unequivocal witness to a sharp division between the methods of these two schools 》 (p. 122) et que le matériel empirique chez Hérophile est l'effet d'une «Aristotelian shadow» (ibid.). Cette précision historique ne change pas le fait que Hérophile pouvait être utilisé par une position sceptique et empirique pour critiquer le dogmatisme, grâce au matériau empirique qu'il présentait, voir par exemple e.g. Hérophile T. $52 ; 53 ; 54$ (Von Staden 1989).

36. Cf. $P H$ I 236 sqq. En réalité il est possible que Sextus cherche à exprimer sa différence avec ses collègues à l'intérieur de l'école empirique, $c f$. Allen 1993, p. 647. Pour une analyse intéressante de la relation entre Sextus et les médecins empiriques, avec une conclusion toute différente, $c f$. Machuca 2008, p. 14 sqq.

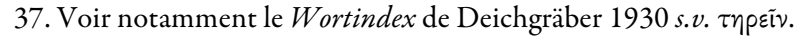


et une méthode. Si la vie quotidienne est une vie sans opinion, c'est précisément parce que les procédures cognitives en jeu dans cette vie fonctionnent sans opinion. Pour Sextus, la vie quotidienne est une vie où l'homme sent, agit, pense, parle d'une façon empirique, c'est-à-dire par imitation des autres, en utilisant sa mémoire et en comparant les situations présentes avec celles du passé38. De la même façon qu'il n'y a aucune nécessité d'avoir une approche théorétique de la grammaire grecque afin d'apprendre le grec et de le parler, en ce qui concerne nos vies, il n'y a aucune nécessité d'une approche théorétique de quoi que ce soit : on peut agir et vivre une vie normale sans théorie. Cette approche pragmatique de la vie est basée sur une forme d'empirisme étroitement liée au thème de la vie quotidienne.

Le texte de $P H$ II 246 invite alors à analyser plus précisément le sens de la $\tau \dot{\eta} p \eta \sigma \varsigma s$ qui détermine l'idée de vie quotidienne. Originairement le mot $\tau \dot{p} p \eta \sigma \varsigma s$ signifie le fait d'observer, de surveiller ou de garder quelque chose dans un contexte politique ${ }^{39}$; mais le terme est fréquemment associé à ż $\mu \pi \varepsilon t-$ pia dans un contexte empirique et signifie « l'observation $»^{40}$. La $\tau \dot{\eta} p \eta \sigma \iota \varsigma$ ne fait pas référence à proprement parler à la vie quotidienne, mais à un fonctionnement cognitif en relation à la vie quotidienne, précisément au fonctionnement empirique qui consiste à observer ce qu'il se passe dans la vie quotidienne. Dans notre contexte $\tau \dot{\eta} p \eta \sigma \iota \zeta$ signifie qu'un sceptique peut expliquer comment la vie donne des principes d'action ${ }^{41}$ : du simple fait qu'ils vivent comme des hommes, avec des compétences naturelles et dans une société, les hommes acquièrent un corpus minimal de connaissances pratiques, ou plutôt de savoir-faire, simplement par l'observation et l'adaptation à leur environnement, et cette aptitude n'est pas particulière à certains mais quelque chose de commun à tous les hommes et lié à leur nature. C'est sur cette dimension que Sextus insiste lorsqu'il emploie dans notre

38. Cf. aussi DL IX 78 qui présente le discours pyrrhonien comme « un souvenir de ce

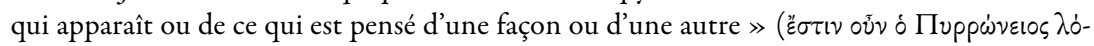

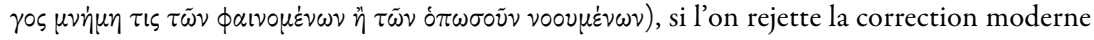

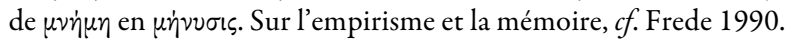

39. Cf. Online Liddell-Scott-Jones Greek-English Lexicon, s.v. $\tau \dot{p} p \eta \sigma \iota s$ qui cite Aristote, Politiques, 1308a30.

40. Chez Galien, voir par exemple la définition de l'expérience dans le De experientia

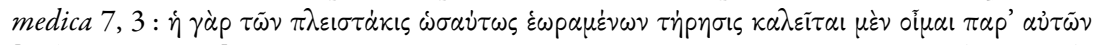

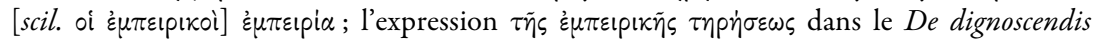
pulsibus libri iv. 8, 857; et De praesagitione ex pulsibus libri, vol. 9, p. 278 qui cite le compte rendu par Hérophile de «some observation and experience rather than teaching a rational

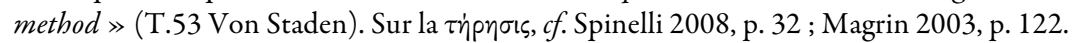

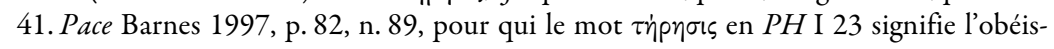
sance à des lois plutôt que l'observation d'objets. 
texte l'expression $\tau \dot{\alpha} \varsigma$ kotvàs $\tau \eta p \dot{\sigma} \sigma \varepsilon ı \zeta$. Ce n'est donc pas un hasard si dans ce

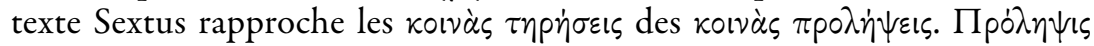
est un terme originairement épicurien ${ }^{42}$. Pour les épicuriens, il s'agit de la condition de la connaissance objective intrinsèquement liée à l'existence d'un objet réel ${ }^{43}$. Mais il semble que Sextus prenne ce terme uniquement pour dire que nous avons tous une forme de pré-connaissance des choses du simple fait que nous vivons, sentons, et pensons. Pour lui, ce qui importe est qu'une $\pi \rho \dot{\partial} \lambda \eta \psi$ is ne soit pas équivalente à une $\kappa \alpha \tau \dot{\alpha} \lambda \eta \psi$ is, à la saisie de la nature des choses ${ }^{44}$. Cela signifie que grâce aux préconceptions, nous pouvons agir dans une société complexe, nous développons des compétences particulières en relation avec notre activité, ou notre intérêt, sans être lié ou engagé dans une théorie.

La position de l'empirisme justifie donc les choix intellectuels faits par Sextus pour penser la vie quotidienne, et éclaire le sens de son concept. Non seulement il s'agit d'une forme de vie particulière, mais il s'agit d'une forme de vie qui ne provient pas d'une décision dogmatique, mais qui se laisse voir empiriquement, dans la vie quotidienne précisément. On pourrait (en tant qu'intellectuel, que philosophe, que critique, et même en tant que critique sceptique) faire l'objection que cette vie n'est pas une vie intéressante parce qu'elle se débarrasse d'une grande partie de ce qui fait l'intérêt de la vie humaine, à savoir les questions intellectuelles et théorétiques. Mais cette restriction est complètement assumée par Sextus comme faisant partie de sa position anti-intellectualiste et anti-philosophique. Le cœur du scepticisme, son but et sa principale référence, ce sont « les besoins de la vie

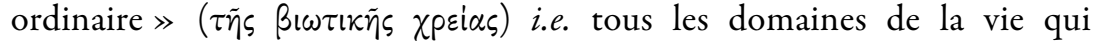
fonctionnent en réalité sans réflexion théorétique ${ }^{45}$; le reste est inutile, de

42. Cf. DL X 33-34.

43. Le fait que les sceptiques utilisent une forme de $\pi p \dot{\partial} \lambda \eta \psi i s$ est confirmé par les passages où Sextus est en accord avec la position d'Épicure selon qui (AM I 57 ; Us. 255) « sans préconception, il n'est possible ni de mener une enquête ni de rencontrer une aporie » (je traduis) bien qu'il refuse l'idée selon laquelle avoir une $\pi p 0 \lambda \eta \dot{\psi} \psi \varsigma$ d'une chose implique de la saisir ( $A M$ VIII, 334a-336a). Voir aussi $A M$ XI 21 et le commentaire ad. loc. de Spinelli 1995.

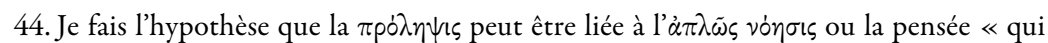
naît à partir de ce qui lui [scil. le sceptique] tombe passivement sous le sens et des raisons qui lui apparaissent d'une manière évidente, cela n'impliquant absolument pas l'existence de ce

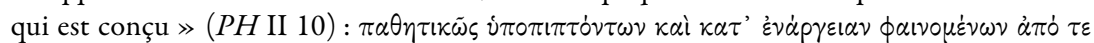

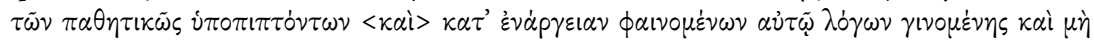

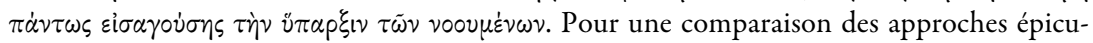

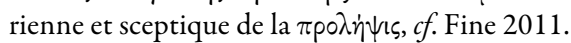

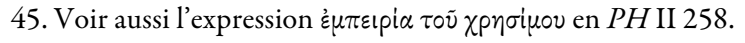


simples mots qui, pour Sextus, sont principalement responsables de nos souffrances $^{46}$.

\subsubsection{Les activités de la vie quotidienne}

En ce qui concerne la variété des activités empiriques, il semble que la vie quotidienne ne soit pas aussi pauvre et uniforme qu'on a pu le dire ${ }^{47}$. Les différences des lois et des traditions expliquent que différentes cultures produisent différents peuples avec une large variété de conceptions et de pratiques. Le processus d'apprentissage cité par PH I 24 explique que différents peuples puissent apprendre et enseigner différents arts et techniques, comme l'astronomie empirique, l'agriculture, la navigation, la grammaire, la médecine, et même la sculpture, la peinture, tous ces processus qui impliquent une grande capacité d'observer et comparer afin d'agir ${ }^{48}$.

L'observation empirique n'est donc pas dogmatique en elle-même, elle est simplement une capacité commune de noter et de se souvenir des $\phi \alpha t-$

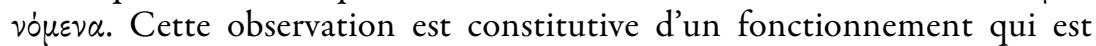
commun, selon Sextus en $A M$ VIII 288, à l'homme et à l'animal :

En ce qui concerne les phénomènes, il [scil. l'animal] a la capacité de tirer des conclusions à partir de l'observation, capacité par laquelle il se rappelle quelles sont les choses qu'il a observées avec lesquelles, lesquelles avant, lesquelles après, et à partir de la rencontre des premières choses il retrouve le reste ${ }^{49}$.

46. Cf. $P H \mathrm{I} 30$.

47. Selon Grgić 2011, p. 74, la liste de PH I 23-24 est « too narrow, for it is obvious that ordinary people are engaged in a much broader range of activities. The point is (...) that typical buman life includes activities - such as cultivating certain virtues, enjoying intimate personal relations, engaging in certain activities exclusively for the sake of pleasure, making new social institutions, creating works of art, etc. - which are not mentioned in the list and it is not even clear what Sextus would make of them ». Néanmoins, le modèle empiriste de la vie quotidienne explique à mon sens une grande partie de ces activités.

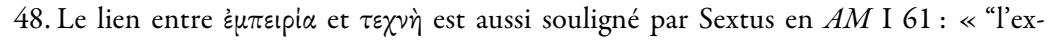
périence" s'applique aussi à l'art comme nous l'avons fait voir dans nos Traités empiriques. Dans la vie quotidienne, on dit indifféremment des mêmes personnes qu'elles sont expé-

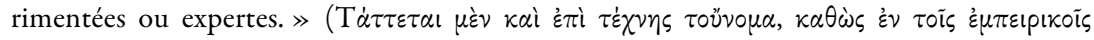

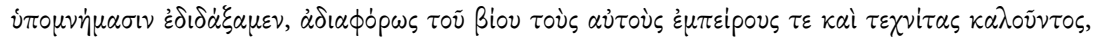
trad. C. Dalimier dans Pellegrin (éd.) 2002 modifiée.)

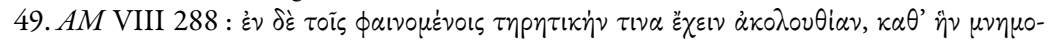

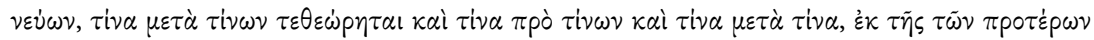
$\dot{v} \pi \circ \pi \tau \dot{\omega} \sigma \varepsilon \omega \varsigma \grave{\alpha} \nu \alpha \nu \varepsilon \circ \tilde{\tau} \tau \alpha \mathrm{l} \tau \dot{\alpha} \lambda$ ol $\pi \dot{\alpha}$. Voir aussi $A M$ VIII 154 pour l'usage de $\sigma \nu \mu \pi \alpha p \alpha \tau \dot{\eta} p \eta \sigma l \varsigma$ dans le contexte de la théorie des signes indicatif et commémoratif. Sur le raisonnement 
Cette capacité d'observer et de lier les phénomènes ensemble peut certainement être liée à la capacité de saisir « la connexion entre les choses » (PH II 236 cité supra). Ces procédures empiriques donnent ainsi une réalité et une substance à la vie sceptique. Ce n'est pas la vie parfois décrite par ceux qui défendent un scepticisme total ou absolu, où le sceptique agirait comme un ordinateur, parlerait comme un perroquet - une vie qui, précisément, paraîtrait absurde au sens commun ${ }^{50}$ - mais la vie vécue selon le sens commun qui conduit à un savoir empirique et non théorétique ${ }^{51}$. Certainement le mot « connaissance » est trop fort et dogmatique; pour cette raison Sextus l'évite, et utilise à la place l'expression $\tau \dot{\eta} p \eta \sigma \iota s$ ż $\pi$ i $\phi a l-$ vou'vols 《l'observation qui s'appuie sur des phénomènes 》 ( $A M \mathrm{~V} 2)$ pour faire référence à cette forme minimale de connaissance que sont « l'agriculture ou la navigation et qui permet de présager sécheresses et inondations, pestes et séismes et autres modifications du même genre du monde qui nous entoure $\gg^{52}$.

Même si nous rappelons les réticences de Sextus vis-à-vis de l'école médicale empirique, même s'il existe des textes qui utilisent des arguments qui ne sont pas toujours favorables à l'ż $\mu \pi \varepsilon ı p^{53} \alpha^{53}$, et même si nous savons que Sextus donne des arguments contre la connaissance sensible avec les dix modes d'Énésidème, la défense et l'usage de tous ces arguments empiriques par Sextus Empiricus ne peuvent pas être méprisés.

Il est donc possible de donner une définition précise de l'expression « vivre selon l'observation de la vie quotidienne » : il s'agit de vivre comme les hommes ordinaires, sans philosophie, sans théorie, sans corpus de connaissances dogmatiques ou scientifiques, mais en utilisant des inférences empiriques, des observations, en utilisant sa mémoire et sa capacité de voir les relations entre les phénomènes, sans se prononcer sur la nature des choses.

associationiste et non inférentiel que permet le signe commémoratif, voir maintenant Tor 2010, p. 71-78.

50. Cette vie caricaturale est caractéristique de l'interprétation « rustique» et radicale du scepticisme.

51. Voir aussi $A M$ VII 158 et $P H$ II 102.

52. Trad. B. Pérez dans Pellegrin (éd.) 2002. Cf. Barnes 1988, p. 71-72, Spinelli 2000 ad loc et Spinelli 2010, p. 258-259.

53. Par exemple en $A M$ VI 32, $c f$. Barnes 1988, p. 70. En ce qui concerne ce passage, il est fort probable d'ailleurs que Sextus se contente de citer un argument d'origine épicurienne contre l'utilité de l'art musical. 
2. La valeur de la vie quotidienne:

en quel sens une philosophie de la vie quotidienne est-elle possible ?

Une fois la fonction et le statut épistémique de la référence à la vie quotidienne clarifiés, il faut étudier son sens philosophique. Ce qui n'est pas aisé à cause d'une véritable ambiguité dans cette référence : en quel sens le projet sceptique est-il celui d'une philosophie de la vie quotidienne? S'agit-il d'une simple description de la vie quotidienne partagée par ceux que nous avons appelés les hommes ordinaires, c'est-à-dire les hommes qui ont un rapport non théorétique aux activités qu'ils mènent ? Ou s'agit-il d'une invitation à vivre conformément à la vie quotidienne, c'est-à-dire un discours de type prescriptif? Si ce projet philosophique qu'est le scepticisme est accompagné d'une recommandation (fût-elle implicite) d'adopter ce genre de vie, nous revenons à une forme de dogmatisme, le dog-

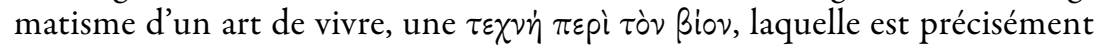
critiquée comme une forme de dogmatisme par Sextus ${ }^{54}$. Mais s'il s'agit d'une pure description, cela remet en question le statut du scepticisme en tant que philosophie, dans la mesure où la description de la vie quotidienne est en soi insuffisante pour constituer un projet philosophique. Pour comprendre - et éventuellement justifier - la cohérence du scepticisme pyrrhonien, il importe de voir comment Sextus échappe à ces deux écueils.

\subsection{La référence à la vie quotidienne peut-elle n'être que descriptive?}

Une interprétation possible du scepticisme et de son usage de la vie quotidienne pourrait être de montrer que Sextus cherche à se limiter à des formulations purement descriptives lorsqu'il dit «nous vivons en observant la vie quotidienne ». Cela serait cohérent avec l'approche empirique précédemment développée : le sceptique suit la vie quotidienne dans laquelle il voit l'homme normal vivant en toute simplicité sans opinion ou croyance dogmatique. Si la vie quotidienne est un concept sceptique, c'est précisément parce qu'il est utilisé non pas comme une construction intellectuelle, selon Sextus, mais comme une simple expérience, l'expérience de ce qui nous est donné à voir sans avoir aucune théorie ou opinion.

Néanmoins, il semble que Sextus ne s'en tienne pas toujours à cette ligne descriptive, et qu'il considère lui-même la possibilité du dogmatisme au sein même de la vie quotidienne :

De là vient que parmi les profanes les uns disent qu'il n'y a qu'un dieu, d'autres plusieurs et différents par leurs formes, de sorte qu'ils tombent 
dans les mêmes suppositions que les Égyptiens, qui estiment que les dieux ont une tête de chien ou une forme de faucon, de bœuf, de crocodile.... ${ }^{55}$

Comme souvent, le cas de la croyance religieuse est intéressant. Même si Sextus a montré que vivre en observant la vie quotidienne permet de sauvegarder quelque chose comme une piété sceptique ${ }^{56}$, cet exemple montre que

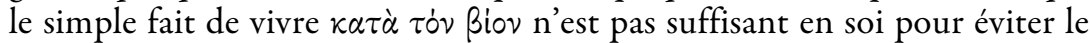
dogmatisme. Au contraire, il semble qu'une proportion significative des gens ordinaires ait des opinions ou des croyances sur des objets cachés, comme par exemple les dieux. Il faut donc concéder que la vie quotidienne sceptique diffère quelque peu des autres vies quotidiennes. Bien sûr, Sextus ne dit pas que toute personne a des croyances, mais le simple fait qu'il

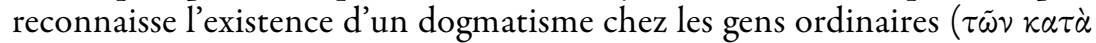
$\tau$ tò Biov) remet en question la valeur univoquement sceptique de la vie quotidienne ${ }^{57}$.

De même, un certain nombre de textes éthiques vont dans la même direction en mettant en lumière une forme de dogmatisme propre aux hommes ordinaires :

En effet si nous laissons de côté les gens ordinaires, parmi lesquels les uns pensent que le bien est le bon état du corps, d'autres la fornication, d'autres la gloutonnerie, d'autres l'ivrognerie, d'autres le jeu de dés, d'autres le lucre, d'autres des choses pires encore... ${ }^{58}$

Les « gens ordinaires » peuvent donc être eux aussi du côté du dogmatisme : ils ont une opinion sur ce qui est naturellement bon ou mauvais ${ }^{59}$. De même, Sextus critique la situation des gens ordinaires qui « se trouvent dans une situation double, du fait des affects eux-mêmes et, dans une mesure qui n'est pas moindre, du fait qu'ils estiment que ces situations sont mauvaises par nature $\gg^{60}$. Dans les deux cas, Sextus oppose clairement

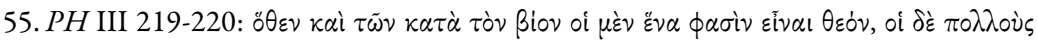

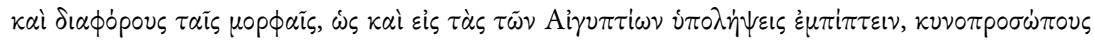

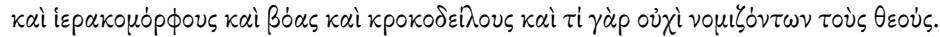

56. Cf. $P H$ I 24 ; III 2 et $A M$ IX 49.

57. Voir aussi $P H$ I 165 et $A M$ VIII 355 pour des exemples d'opposition entre la vie quotidienne et des positions philosophiques comme deux formes de dogmatisme.

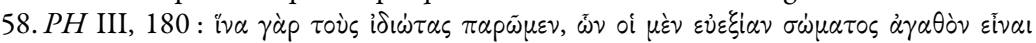

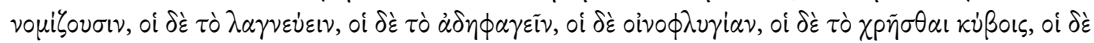

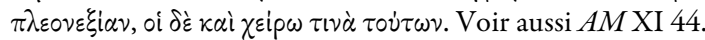

59. Cf. Bett 2011, p. 7 et p. 10 , n. 11 pour l'hypothèse intéressante selon laquelle « concerning matters of good or bad (unlike many others matters that are solely the provinces of theorists), ordinary people also have a reflective side ».

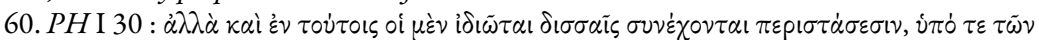

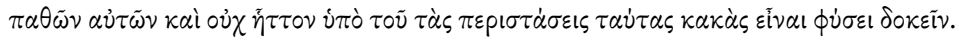


l'« homme ordinaire » et le sceptique, en plaçant clairement le premier du côté du dogmatisme. Certes, le terme employé dans ces deux derniers textes ne renvoie pas au ßios mais à l'io $\delta \dot{\omega} \tau \eta$ s, i.e. à l'homme du commun, « celui qui n'a pas de connaissance professionnelle » comme le dit le Liddell and Scott ${ }^{61}$. Mais il semble bien que cela revienne au même : l'homme ordinaire, c'est-à-dire dans le contexte de ces textes, l'homme qui n'adhère pas à une conception philosophique du bien ou du mal, vit finalement comme un philosophe dogmatique, du moins en ce qui concerne ses jugement moraux. Suivre la voie sceptique - au moins d'un point de vue moral - ne peut donc pas consister à revenir à la vie normale et quotidienne puisque de ce point de vue, elle est susceptible de verser dans le dogmatisme.

Donc, même si l'option purement descriptive pourrait constituer une lecture satisfaisante en ce qui concerne la cohérence du projet de Sextus, cette option n'est pas tenable jusqu'au bout. La vie quotidienne dont parle Sextus reste une vie quotidienne particulière : peut-être la relation particulière que Pyrrhon avait avec la quotidiennetée ${ }^{62}$, peut-être la vie vécue par quelques médecins particuliers ${ }^{63}$, mais pas la simple et pure description de la vie quotidienne $e^{64}$. Il faut donc que la référence à la vie quotidienne contienne une dimension prescriptive. En outre, ce qui est perdu ici en scepticisme, pour ainsi dire, permet de sauver le statut philosophique du scepticisme. En constituant une forme particulière de vie quotidienne comme point de référence, Sextus est cohérent avec ce qui est attendu d'un philosophe, du moins d'un philosophe hellénistique, peut-être même d'un philosophe antique en général : produire des prescriptions en vue d'une vie meilleure.

\subsection{La vie quotidienne comme une référence prescriptive}

Lorsque Sextus cherche à décrire la particularité de la philosophie sceptique, il utilise des termes qui visent à la distinguer de la pratique dogmatique de la philosophie, en la désignant comme une voie, une à $\gamma \omega \gamma \grave{\eta}(P H$

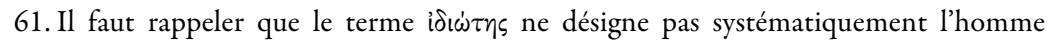
ordinaire en tant qu'il est dogmatique. Le terme peut aussi désigner la même chose que la vie quotidienne et marquer une différence entre les hommes communs et les philosophes (cf. e.g. III $243-244 ; 249$ ), preuve supplémentaire, s'il en est besoin, que l'usage des concepts par Sextus n'est pas systématique.

62. Parce que certains témoignages sur Pyrrhon montrent qu'il menait une vie simple. Cf. T. 14 DC (apud DL IX 66) ; T. 18. C'est ainsi que Grgić 2011 comprend la référence à la vie quotidienne, $c f$. p. 70-71.

63. Cf. e.g. Galien, Subfiguratione empirica, chap. 9 (p. 82 Deichgräber 1930 ; T. 67 Decleva Caizzi 1981).

64. Cf. Thorsrud 2003, p. $234:$ : Sextus is not a champion of ordinary life in its undeveloped form. The skeptical life is an achievement and not merely the recovering of native innocence lost to philosophical speculation. » 
I 4). La philosophie sceptique comporte néanmoins quelques caractéristiques communes avec la philosophie dogmatique. Sextus veut à la fois être distingué des philosophes dogmatiques, et être reconnu et lu par des philosophes. Pour ces raisons, Sextus demande si le sceptique appartient à une école en $P H$ I 16-17 :

Si l'on dit qu'une école est une inclination à suivre beaucoup de dogmes qui se suivent les uns les autres aussi bien qu'ils suivent les choses apparentes, et si l'on dit qu'un dogme est l'assentiment à quelque chose d'obscur, alors nous dirons que le sceptique n'appartient pas à une école. Mais si l'on déclare qu'une école est la voie qui suit un raisonnement déterminé en accord avec l'apparence, ce raisonnement étant celui qui montre comment il semble possible de vivre correctement - nous prenons « correctement 》 non seulement au sens de « selon la vertu» mais en un sens plus large - et qui tend à nous donner la possibilité de suspendre notre assentiment, nous disons qu'il appartient à une école. Car nous suivons un raisonnement déterminé qui nous montre, en accord avec l'apparence, comment vivre selon les coutumes traditionnelles, les lois, les modes de vie et nos affects propres $^{65}$.

Comme Roberta Ioli le fait remarquer, il s'agit du seul texte où Sextus décrit le scepticisme comme une aipeбıs; la plupart du temps il préfère $\dot{\alpha} \gamma \omega \gamma \dot{\eta}^{66}$. Et même dans ce texte, il affaiblit la connotation dogmatique de l'aipeбıs, qui désigne d'abord un choix systématique d'un corpus de thèses philosophiques à suivre dans sa vie. Le problème est que même dans le cas

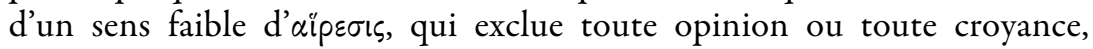
Sextus en revient à une conception classique de la philosophie, c'est-à-dire à un discours ou à un argument ( $\lambda$ óyos) qui montre une forme de modèle de vie, d'idéal, ou de norme ${ }^{67}$. La volonté de Sextus est bien d'affaiblir ces

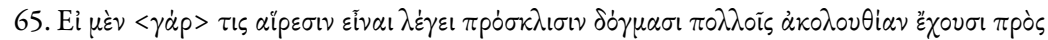

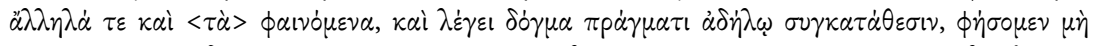

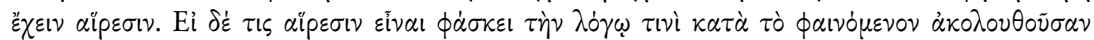

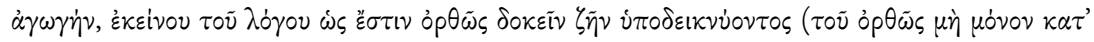

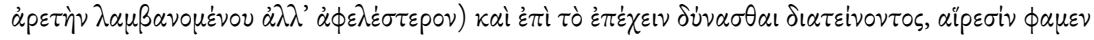

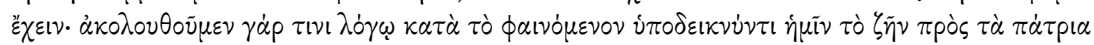

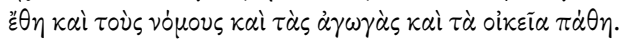

66. Cf. Ioli 2003, p. 403 sqq. Sur l'usage pyrrhonien d' $\alpha \hat{\gamma \omega \gamma} \dot{\eta}, c f$. aussi DL I, 20 ; Aristoclès, apud Eusèbe, PE XIV 18, 30 et Photius, Bibl. 212, 170b2. Cette terminologie a été étudiée par Glucker 1978, p. 165 sqq.

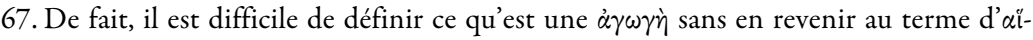

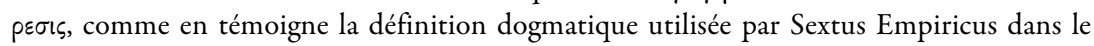
contexte du dixième trope, en $P H$ I 145: « un mode de vie est un choix de vie ou d'activité

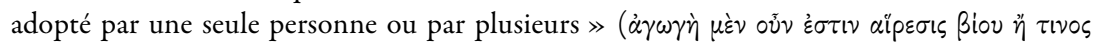

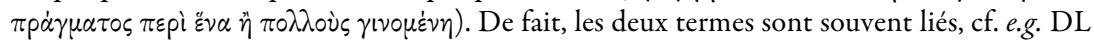
I, 19. 
termes en corrigeant les mots mêmes de la philosophie : ce qu'il fait précisément dans ce texte quand il évite le terme àpet⿳亠 et quand il dépeint son propre discours sceptique comme le récit d'une expérience personnelle et non un moyen universel et systématique d'être heureux. Il n'en reste pas moins qu'il doit présenter sa propre pratique comme philosophique, au moins pour ne pas tomber sous l'objection selon laquelle le scepticisme n'est pas une philosophie ${ }^{68}$. Donc le scepticisme est une philosophie, c'est le choix d'une certaine forme de vie présentée comme un contre-modèle pour ceux qui souffrent du dogmatisme ${ }^{69}$. Le fait que ce modèle soit emprunté à la vie quotidienne ne change rien à la situation. Le sceptique cherche à avoir une vie qui se rapproche de la forme de vie qui est reconnue comme bonne, du moins comme meilleure que la vie dogmatique.

On peut enfin noter que cette interprétation suppose elle aussi que la vie quotidienne dont parle Sextus n'est pas tout à fait la vie de l'homme du commun, mais bien une certaine forme de vie quotidienne, la vie quotidienne sceptique. De toute façon il semble assez naif de faire de la vie quotidienne la norme de la vie bonne : comment pourrions-nous revenir à une relation simple, nue à la vie, une fois abandonnée cette simplicité ? Et pouvons-nous même décider de vivre simplement ? En réalité, ce qui fait la vie quotidienne, c'est précisément sa spontanéité, et non pas le résultat d'un effort, encore moins d'une éthique. Le philosophe qui cherche à vivre selon la pure et simple vie quotidienne serait dans la même situation que l'homme qui, pour oublier, doit se souvenir d'oublier, un peu comme lorsque nous essayons de suivre des impératifs comme «soyez naturel », ou « calmez-vous! 》

$\mathrm{Si}$ « vivre suivant la vie quotidienne » signifie « vivre suivant la vie quotidienne comme elle était vécue par Pyrrhon », ou par n'importe qui ayant vécu sans opinion et empiriquement, le modèle est probablement moins naif ${ }^{70}$. Et Sextus maintient ainsi un équilibre qui lui permet d'être intégré dans le chœur philosophique (pour être lu comme un philosophe,

68. Pour ce type d'objections, voir e.g. Aristoclès apud Eusèbe, PE XIV 18, 30.

69. Même Ioli 2003, p. 415, malgré sa défense élégante de la cohérence de la position de

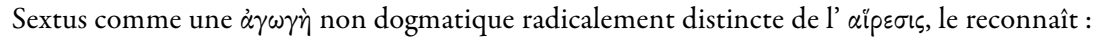
«Sceptics are philosophers as long as they cohere, albeit involuntarily, with an ephectic logos, that is, they do not assent to what appears existent and suspend judgment. (...) By following such a logos they make a kind of theoretical choice (however undogmatic and uncommitted it may be), which is consistent with the ephectic character of the logos itself. »

70. Tel est peut-être le sens de la formule sibylline que l'on trouve en $P H$ I 7 : « la voie sceptique est appelée (...) "pyrrhonienne" du fait qu'il nous semble que Pyrrhon s'est approché du scepticisme d'une manière plus consistante et plus éclatante que ceux qui l'ont

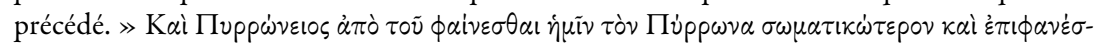

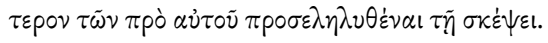


écouté par des oreilles philosophiques, participer aux discussions philosophiques) sans pour autant tomber dans le dogmatisme.

On peut, certes, passer outre ces objections en arguant que l'exposé de Sextus au début des Esquisses est trop académique ou scolaire pour être

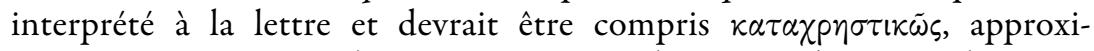
mativement, parce que les sceptiques ne veulent pas se battre sur les mots, et utilisent les concepts de la philosophie dans l'état où ils les trouvent ${ }^{71}$. Mais il n'est pas sûr que cela soit un bon argument pour répondre au fait que Sextus, volens nolens, fait une proposition éthique dans son œuvre, quand bien même cette proposition reposerait sur des bases empiriques et pragmatiques.

\subsection{Le paradoxe du retour à la vie quotidienne}

Nous sommes donc en présence de deux interprétations également possibles et justifiables mais posant également des problèmes de cohérence avec le projet sceptique dans son ensemble. Faut-il en conclure une fois encore à la schizophrénie de Sextus ou, plus prosaïquement, à son incohérence ? Pour conclure, je voudrais défendre la cohérence de la position de Sextus et réaffirmer la centralité du concept de vie quotidienne.

Pour résumer, nous avons vu que Sextus veut à la fois utiliser les vertus prescriptive et descriptive de la référence à la vie quotidienne. La vertu descriptive est qu'elle permet un lien direct vers une voie empirique de justification sans que le sceptique ne soit engagé à aucune opinion; la vertu prescriptive est qu'elle donne un sens éthique à la philosophie sceptique. Ces deux options ne paraissent pas compatibles, du moins intuitivement. Mais c'est ce dernier point qu'il faut maintenant discuter en montrant que chez Sextus il n'y a rien qui rende ces deux options exclusives et qu'elles pourraient donc bien être complémentaires.

Pour comprendre cet aspect, il convient de reprendre la comparaison médicale utilisée par Sextus : le dogmatisme est une maladie. Mais à quoi s'oppose cette maladie ? En quoi consiste alors la santé ? Pour répondre à cette question, il faut distinguer deux possibilités : soit le scepticisme, soit la vie quotidienne telle qu'elle est décrite par Sextus lorsqu'il parle de la vie avant le dogmatisme. Il faut donc distinguer en quelque sorte deux vies quotidiennes : la vie avant le dogmatisme, la vie rêvée, où il n'est pas besoin de médecine, de traitement, ni de norme ou d'idéal d'un côté, et de l'autre

71. Cf. $A M$ I 233-234: «En conséquence, en philosophie, nous nous alignerons sur l'usage de philosophes, en médecine sur l'usage le plus médical, et dans la vie courante, sur

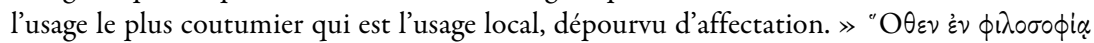

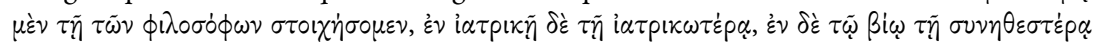

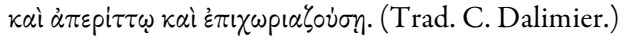


la vie après le dogmatisme, c'est-à-dire la vie qui a perdu un certain rapport à la vie. Dans le premier cas, nul besoin d'atteindre « la tranquillité en matière d'opinions » parce qu'il n'y a, par hypothèse, pas d'opinion du tout, mais une relation directe à la vie et à ce qui lui est utile, il n'y a que la nécessité d'obtenir « la modération des affects dans les choses qui s'imposent à nous $\gg^{72}$. Dans le second cas, cependant, les opinions et les croyances sont devenues partie prenante de notre réalité, et nous avons besoin selon Sextus de nous battre contre ces opinions afin d'atteindre « la tranquillité en matière d'opinion » en plus de « la modération des affects dans les choses qui s'imposent à nous $\gg$.

La question devient donc de savoir comment on en est arrivé à ne pas vivre « en suivant la vie quotidienne ». Comment peut-on en venir à nier l'évidence, à transformer la réalité et à perdre la dimension commune et naturelle de l'expérience quotidienne ? Le dogmatisme est une anomalie, une perversion de la pensée qui coupe artificiellement le philosophe ou l'homme de science de cette expérience commune. Il y a des signes chez Sextus qui indiquent cette ligne de pensée. C'est le dogmatisme qui coupe le dogmatique de la réalité, de l'expérience des $\phi \alpha \iota v \rho \dot{\varepsilon}^{2} \nu \alpha^{73}$. C'est d'ailleurs ce qui fait que, contre toute attente, le sceptique peut lui-même se retrouver en situation d'avoir à argumenter contre le фaเvó $\mu \varepsilon v o v$ :

Si nous proposons des arguments même directement contre les phénomènes, nous ne proposons pas ces arguments dans l'intention de rejeter les phénomènes, mais pour bien montrer la précipitation des dogmatiques; car si le raisonnement est trompeur au point qu'il s'en faille de peu qu'il ne dérobe même les phénomènes sous nos yeux, combien ne faut-il pas se défier de lui dans le cas des choses obscures, pour que nous ne soyons pas entraînés par lui à nous précipiter ${ }^{74}$ ?

La précipitation $(\dot{\eta} \pi p \circ \pi \varepsilon \dot{\tau} \varepsilon 1 \alpha)$ des dogmatiques est la preuve de leur cécité même en ce qui concerne ce qui est évident. Aveuglé par ses croyances, le dogmatique tourne le dos à la réalité en rejetant les phénomènes qui pourtant sont évidents. De la même façon, on peut penser que le dogmatique a perdu quelque chose de sa relation à la vie quotidienne. Et c'est à cause de l'existence d'un dogmatisme qui tourne le dos à la vie quotidienne que les sceptiques doivent présenter un modèle pour l'action qui met la vie

72. Suivant la terminologie de $P H$ I 25.

73. Cf. $P H \mathrm{I} 30$.

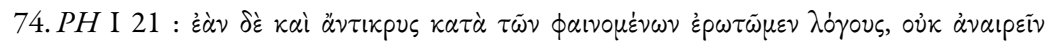

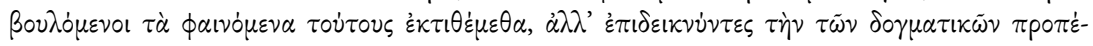

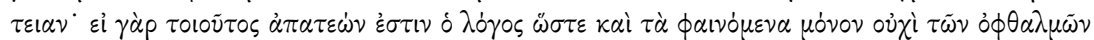

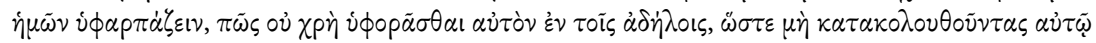
$\pi \rho \circ \varepsilon \tau \varepsilon \dot{\varepsilon} \varepsilon \sigma \alpha l$; 
quotidienne au centre. Pire, Sextus parle à une société qui est massivement prise dans le dogmatisme en ce qui concerne les questions morales, et où l'homme du commun partage avec les philosophes et les hommes de science des opinions, des croyances et des thèses.

Il est donc possible que ce dilemme corresponde finalement à deux situations : la situation normale, saine (qui est une forme d'idéal perdu) et la situation dogmatique. La situation dogmatique est de toute évidence la plus répandue, mais le but de Sextus est de témoigner qu'une autre vie est possible, sur la base de l'expérience nue de la vie. Ce témoignage sert donc à définir une nouvelle forme de vie, une vie sceptique et empirique.

Récapitulons maintenant quelques points de cette étude sur la vie quotidienne dans le scepticisme de Sextus.

- La vie quotidienne est un concept sceptique original non emprunté aux dogmatiques.

- Même si ce concept est plutôt utilisé dans des situations dialectiques

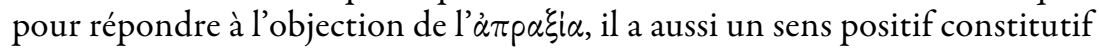
de la définition du mode de vie sceptique.

- Le statut empirique de la vie quotidienne donne un contenu à cette vie, sans emprunter aucune thèse dogmatique. De nombreuses assertions de Sextus sur la vie quotidienne sont descriptives.

- Cependant une philosophie sceptique ne peut se contenter d'une description. Comme le dit Roberta Ioli, « everybody can follow customs, laws, his own affections and natural needs without becoming, simply for that reason, a philosopher $\gg{ }^{75}$. Donc les sceptiques doivent inventer et promouvoir une nouvelle relation à la vie quotidienne, qui ne peut pas être considérée comme un simple retour à la vie quotidienne, mais comme un projet pour une vie anti-intellectuelle et anti-théorétique.

- En soi le scepticisme exprime une tentative radicale de s'extraire de la philosophie, mais il est forcé de le faire à partir de tous les termes, problèmes et caractéristiques définis par la tradition sceptique. Malgré l'originalité radicale du projet, Sextus est conscient qu'il parle et écrit pour des dogmatiques, c'est-à-dire pour des gens qui ont déjà perdu leur relation naturelle et non dogmatique à la vie quotidienne, qui vivent dans un monde qui n'est pas constitué que de faits mais aussi d'opinions.

Il faut maintenant revenir à notre question première: sur quel fondement s'appuie l'appel à la vie quotidienne de Sextus Empiricus ? Dans la philosophie contemporaine, deux options ont été choisies pour penser la vie quotidienne : la sociologie ou la phénoménologie. Avec Sextus, apparaît une troisième option : le scepticisme, mais un scepticisme où l'expérience est le mot clef. Sur la base de l'expérience commune de la vie, Sextus pense 
que nous, les philosophes, nous pouvons construire une relation plus naturelle avec nos propres vies ${ }^{76}$.

76. Cet article a fait l'objet d'un certain nombre de communications et de discussions qui en ont changé considérablement l'orientation et m'ont permis de l'améliorer. Je remercie à cette occasion T. Bénatouil, R. Bett, A. Correa Motta, L. Corti, A. Lozano Vasquez, D. Machuca, P.-M. Morel, ainsi que les deux commentateurs anonymes de Philosophie antique. 


\section{BIBLIOGRAPHIE}

Allen, J. 1993 : « Pyrrhonism and medical Empiricism : Sextus Empiricus on Evidence and Inference », Aufstieg und Niedergang der Römischen Welt, II, 37, 1, Berlin, 1993, p. 646-690.

ANNAS, J. \& J. BARNES 1994 : Sextus Empiricus: Outlines of Scepticism, Cambridge/New York, 1994.

BARNES, J. 1988 : « Scepticism and the Arts », dans R. J. Hankinson (éd.), Method, Medicine and Metaphysics: Studies in the Philosophy of Ancient Science, Edmonton, 1988, (=Apeiron, $21.2[1988])$, p. 53-77.

- 1997 : « The beliefs of a Pyrrhonist », dans M. Burnyeat et M. Frede (éd.), 1997, p. 5891.

BÉGOUT, B. 2005 : La Découverte du quotidien, Paris, 2005.

BETT, R. 1989 : « Carneades' pithanon : a Reappraisal of its Role and Status », Oxford Studies in Ancient Philosophy, 7 (1989), p. 59-94.

- 1993: «Scepticism and Everyday Attitudes in Ancient and Modern Philosophy», Metaphilosophy, 24/4 (1993), p. 363-381.

- 2010 (éd.) : The Cambridge Companion to Ancient Scepticism, Cambridge, 2010 (Cambridge Companions to Philosophy).

- 2011 : « How Ethical Can an Ancient Skeptic Be? », dans Diego E. Machuca (éd.), Pyrrhonism in Ancient, Modern, and Contemporary Philosophy, Dordrecht, 2011 (The New synthese historical Library, 70), p. 3-17.

Brochard, V. 1887 : Les Sceptiques grecs, Paris, 1887 [réimpr. Paris, Livre de poche, 2002].

BRUNSCHWIG, J. 1999 (trad.) : Diogène Laërce. Vies et doctrines des philosophes illustres, livre IX : Pyrrhon, dans M.-O. Goulet-Cazé (éd.), Diogène Laërce. Vies et doctrines des philosophes illustres, Paris, 1999 (Le Livre de Poche), p. 1099-1138.

Burnyeat M. \& M. Frede 1997 (éd.) : The Original Sceptics: A Controversy, Indianapolis, 1997.

BURY, R. G. 1933 (trad.) : Sextus Empiricus. Outlines of pyrrhonism, London, 1933 (The Loeb Classical Library. Greek authors, 273).

CHIESARA, M. L. 2001 (éd. et trad.) : Aristocles of Messene. Testimonia and fragments, Oxford/New York, 2001 (Oxford Classical Monographs).

DALIMIER, C. 1991 : «Sextus Empiricus contre les grammairiens : ce que parler grec veut dire », dans Suzanne Said (éd.), Hèllènismos : quelques jalons pour une histoire de Pidentité grecque, actes du Colloque de Strasbourg, 25-27 octobre 1989, Leyde/New York, 1991 (Travaux du centre de recherche sur le Proche-Orient et la Grèce antique, 11), p. 17-32.

Decleva Caizzi, F. 1981 (éd. et trad.) : Pirrone. Testimonianze, Napoli, 1981 (Elenchos, 5).

DeICHGRÄBER, K. (éd.) 1930 : Die griechische Empirikerschule : Sammlung der Fragmente und Darstellung der Lehre, Berlin/Zürich, 1930.

EsTIENNE, H. 1562 : Sexti philosophi Pyrrhoniarum hypotypōseōn libri III : quibus in tres philosophiae partes severissime inquiruntur: Libri magno ingenii acumine scripti, variáque doctrina referti : Graecè nunquam, Latinè nunc primùm editi, [Genève], Excudebat idem Henricus Stephanus, 1562.

FINE, G. 2011 : « Concepts and inquiry: Sextus and the Epicureans », dans B. Morrison \& K. Ierodiakonou (éd.), Episteme, etc. : Essays in Honour of Jonathan Barnes, Oxford, 2011, p. 90-114.

FrEDE, M. 1990 : «An Empiricist View of Knowledge: Memorism », dans S. Everson (éd.), Companions to Ancient Thought 1 : Epistemology, Cambridge, 1990 (Companions to Ancient Thought), p. 225-250. 
- 1997 : « The Sceptic’s Beliefs », dans M. Burnyeat \& M. Frede (éd.), 1997, p. 1-24.

Gallego CaO, A. et T. MuÑoz Diego 1993 (trad.) : Sextus Empiricus. Esbozos Pirrónicos, Madrid, 1993 (Biblioteca Clásica Gredos, 179).

GLUCKER, J. 1978 : Antiochus and the late academy, Göttingen, 1978 (Hypomnemata, 56).

GrGIĆ, F. 2011 : «Skepticism and Everyday Life », dans Diego E. Machuca (éd.), New Essays on Ancient Pyrrhonism, Leyde/Boston, 2011 (Philosophia antiqua, 126), p. 69-90.

IOLI, R. 2003: «Agoge and related Concepts in Sextus Empiricus», Siculorum Gymnasium, N.S. 56 (2003), p. 401-428.

IOpPolo, A. M. 1986 : Opinione e scienza : il dibattito tra Stoici e Accademici nel III e nel II secolo a. C., Napoli, 1986 (Elenchos, 12).

- 2009: La testimonianza di Sesto Empirico sull Accademia scettica, Napoli, 2009 (Elenchos, 53).

Kudlien, F. 1964: « Herophilus und der Beginn der medizinischen Skepsis », Gesnerus, 21 (1964), p. 1- 13.

Lefebvre, H. 1958 : Critique de la vie quotidienne, Paris, 1958 (Le sens de la marche).

MACHUCA, D. 2008 : « Sextus Empiricus : His Outlook, Works, and Legacy », Freiburger Zeitschrift für Philosophie und Theologie, 55/1 (2008), p. 28-63.

MAGRIN, S. 2003 : Scetticismo e fenomeno in Sesto Empirico : la critica ai grammatici e ai retori, Napoli, 2003 (Saggi Bibliopolis, 77).

MATES, B. 1996 (trad.) : Sextus Empiricus. The Skeptic Way : Sextus Empiricus's Outlines of Pyrrhonism, New York, 1996.

ObdrZaleK, S. 2012 : « From Skepticism to Paralysis : The Apraxia Argument in Cicero's Academica », Ancient Philosophy, 32/2 (2012), p. 369-392.

Pappenheim, E. 1877 (trad.): Sextus Empiricus. Des Sextus Empiricus Pyrrhoneische Grundzüge, Leipzig, 1877 (Philosophische Bibliothek, 74).

Pellegrin, P. 1997 (trad.): Sextus Empiricus. Esquisses pyrrhoniennes, Paris, 1997 (Points. Essais, 352).

- 2002 (éd.) : Sextus Empiricus. Contre les professeurs, trad. de C. Dalimier, D. Delattre, J. Delattre et al., Paris, 2002 (Points. Essais, 489).

SEdley, D. N. 1977 : « Diodorus Cronus and Hellenistic Philosophy », Proceedings of the Cambridge Philological Society, N.S. 23 (1977), p. 74-120.

SPINELLI, E. 1995 (éd. et trad.) : Sextus Empiricus. Contro gli etici, Napoli, 1995 (Elenchos, 24).

- 2000 (éd. et trad.) : Sextus Empiricus. Contro gli astrologi, Napoli, 2000 (Elenchos, 32).

- 2005: Questioni scettiche : letture introduttive al pirronismo antico, Roma, 2005 (I saggi, $34)$.

- 2008: «Sextus Empiricus, l'expérience sceptique et l'horizon de l'éthique », Cabiers philosophiques, 115 (2008), p. 29-45.

-2009: «Dei sofismi : Sesto Empirico, gli eccessi della logica dogmatica e la vita comune », dans W. Lapini, L. Malusa \& L. Mauro, Gli antichi e noi: Scritti in onore di Antonio Maria Battegazzore, Genova, 2009, p. 239-253.

- 2010 : «Pyrrhonism and the Specialized Sciences », dans R. Bett 2010 (éd.), p. 249264.

STRIKeR, G. 1980 : «Sceptical Strategies », dans M. Schofield, J. Barnes et M. Burnyeat (éd.), Doubt and Dogmatism: Studies in Hellenistic Epistemology, Oxford, 1980, p. 54-83.

TeSCARI, O. 1926 (éd. et trad.) : Sextus Empiricus. Schizzi pirroniani, con Paggiunta dei passi paralleli di Sesto stesso, di Diogene Laerzio, di Filone e di altri, Bari, 1926.

ThORSRUD, H. 2003 : « Is the Examined Life Worth Living? A Pyrrhonian Alternative », Apeiron: A Journal for Ancient Philosophy and Science, 36/3 (2003), p. 229-249. 
TOR, S. 2010 : « Argument and Signification in Sextus Empiricus: Against the Mathematicians VIII. 289-290 », Rhizai. A Journal for Ancient Philosophy and Science (2010), p. 63-90.

VOGT, K. M. 2010 : «Scepticism and Action », dans R. Bett 2010 (éd.), p. 165-180.

VON STADEN, H. 1989 (éd. et trad.) : Herophilus. The Art of Medicine in Early Alexandria : Edition, Translation and Essays, New York, 1989. 